\title{
Einfluss des nach der TOBEC-Methode bestimmten Fettgehaltes bei Kaninchen auf die Wurfleistungen und die Körperzusammensetzung der Nachkommen
}

\begin{abstract}
Summary
Title of the paper: Effect of selection on body fat content by means of the TOBEC method on some reproductive traits of rabbit does and on the body composition of growing rabbits

In this experiment body fat content of Pannon White growing rabbits was determined at 10 weeks of age using an EM-SCAN SA-3152 type Small Animal Body Composition Analyser (TOBEC method). Based on the fat content determined the best and worst $16 \%$ of the does and the best and worst $8 \%$ of the bucks were chosen and mated with each other (fatty doe with fatty buck and lean doe with lean buck). It was found that the conception rate was significantly higher and the number of inseminations needed for the second kindling significantly lower in the case of fatty rabbits. An important, but not significant difference was observed in the case of total litter size at birth, which decreased in the case of live born litter size. This reason was the significantly higher ratio of the dead born pups in the case of non-fatty rabbits. Due to the higher ratio of total litter loss and suckling mortality in the case of fatty rabbits the litter size at 21 days differed notably, but again not significantly. In the offsprings of the $F_{1}$ generation it was found that the fat content estimated and also the ratio of scapular and abdominal fat to the live weight calculated differed significantly $(\mathrm{P}<0.05)$ from each other in the two experimental groups. The estimated fat content was $42 \%$ higher in the offsprings of fatty than in the offsprings of non-fatty rabbits. The differences in the ratio of the scapular and abdominal fat content to the live weight were $26 \%$ and $51 \%$, respectively.
\end{abstract}

Key Words: rabbit, selection, TOBEC, body composition, fat, reproduction traits

\section{Zusammenfassung}

Es wurde der Fettgehalt von lebenden 10 Wochen alten Kaninchen der Rasse Pannon-Weiße mit Hilfe der TOBEC Methode bestimmt. Auf Grund der geschätzten Werte wurden von den weiblichen Tieren jeweils die extremen $16 \%$, von den männlichen Tieren die extremen $8 \%$ zur Weiterzucht ausgewählt. Die ausgewählten fetten Mütter wurden mit Sperma von fetten Rammlern besamt, die mageren Mütter dagegen mit Sperma der mageren Rammler. Es wurde festgestellt, dass die Trächtigkeitsrate bei den fetten Häsinnen signifikant besser, die Zahl der nötigen Besamungen zum zweiten Wurf signifikant kleiner war. Ein deutlicher, aber statistisch nicht bewiesener Unterschied bestand in der Anzahl insgesamt geborener Jungtiere, der Unterschied bei den lebend geborenen war geringer. Die Ursache dafür lag im signifikanten Unterschied zwischen dem Anteil der tot geborenen Jungen zwischen fetten und mageren Häsinnen. Auf Grund des größeren Anteils von Wurfausfällen und der höheren Mortalität von Jungen in der Säugezeit bei den fetten Häsinnen, ergab sich ein höherer, aber nicht signifikanter Unterschied in der Wurfgröße am 21. Lebenstag. Bei den Nachkommen der $F_{1}$ Generation hat sich der geschätzte Fettgehalt und auch der Anteil des Nacken- und Nierenfettes gegenüber dem Schlachtgewicht als statistisch signifikant zwischen den beiden Versuchsgruppen erwiesen. Der geschätzte Fettgehalt bei den Nachkommen der fetten Tiere war $42 \%$ höher als bei Nachkommen der mageren Tiere. Der Unterschied zwischen den beiden Gruppen im Anteil des Nacken- und Nierenfettes zum Schlachtgewicht lag bei $26 \%$ bzw. $51 \%$.

Schlüsselwörter: Kaninchen, Selektion, TOBEC, Körperzusammensetzung, Fett, Reproduktionsmerkmale

\section{Einleitung}

Zur Bestimmung der Körperzusammensetzung des Kaninchens sind in der Praxis mehrere Verfahren verbreitet (FEKETE, 1992). Von diesen Verfahren werden sowohl in 
der Forschung als auch in der praktischen Tierzucht Schlachtungen und chemische Ganzkörperanalysen am häufigsten angewandt. Diese Methoden haben aber trotz ihrer Genauigkeit den Nachteil, dass durch die Schlachtung der Tiere, diese als potentielle Zuchttiere nicht mehr verfügbar sind und die Ergebnisse nur aus der Nachkommenoder Geschwisterprüfung zur Verfügung stehen (SZENDRÖ et al.). Dieser Umstand führte seit Jahrzehnten bei Nutztieren zur Entwicklung von Methoden die eine Bewertung der Zusammensetzung des Schlachtkörpers ,in-vivo“ mit der nötigen Genauigkeit ermöglichen.

So bemüht sich seit Beginn der 50er Jahre die Tierzuchtwissenschaft um den Einsatz von Ultraschall- und Röntgenverfahren (KALLWEIT et al., 1994; ROSNER et al., 2000) Von den sogenannten non-invasiven Verfahren bedeuten Computer Tomographie (CT) und Nuklearmagnetische Resonanz Tomographie (MR) die heutige Spitzentechnologie. Mit beiden Methoden wurden zahlreiche Untersuchungen durchgeführt um den Fett- und Muskelgehalt lebender Nutztiere zu bestimmen (SKJERVOLD et al., 1981; VANGEN, 1984; BENTSEN et al., 1986; KALLWEIT et al., 1994; SCHOLZ et al., 1995). An der Universität Kaposvar wurden diese Methoden u.a. auch erfolgreich bei Kaninchen sowohl zur Bestimmung des Schlachtwertes und der Körperzusammensetzung mit der nötigen Genauigkeit (SZENDRÖ et al.; ROMVÁRI, 1996; KÖVÉR et al., 1998; MILISITS, 1998) als auch zur Darstellung der Veränderungen der Körperzusammensetzung bei lebenden Kaninchen (MILISITS, 1998; MILISITS et al., 1999a, 1999b) genutzt. Als Nachteil beider Methoden müssen aber die hohen Investitions- und Betriebskosten, sowie der feste Sitz der technischen Anlagen erwähnt werden, was in vielen Fällen den Transport der Versuchstiere über grössere Entfernungen und damit unnötigen Stress bedeutet.

Eine mögliche Lösung für dieses Problem kann das EM-SCAN-Gerät (TOBEC-Methode) sein, das wegen seiner Abmessungen einen mobilen Einsatz ermöglicht. Als weiterer Vorteil des Gerätes erweisen sich die erheblich geringeren Investitions- und Betriebskosten gegenüber dem Einsatz von $\mathrm{CT}$ und MR.

Das TOBEC-Verfahren, welches ursprünglich für die Kinderheilkunde entwickelt wurde, dient in erster Linie zur Bestimmung der fettfreien Substanz des Körpers. Dieses Gerät kann auch zur Feststellung der Körperzusammensetzung von leichtgewichtigen Nutz- und Labortieren (FEKETE et al., 1995) eingesetzt werden. Die bisherigen Ergebnisse bewiesen, dass diese Methode zur Feststellung des Gewichtes des fettfreien Körper mit hoher Genauigkeit $(r=0,88-0,99)$ geeignet ist (CUNNINGHAM et al., 1986; FIOROTTO et al., 1987; FEKETE und BROWN, 1993; STAUDINGER et al., 1995). Bei der Schätzung des Fettanteils ergab sich dagegen nur eine mittlere Verlässlichkeit bei Kaninchen ( $\mathrm{r}=0,59$; FEKETE et al., 1995).

Da für die Praxis die Bestimmung des Fettgehaltes viel wichtiger ist, haben sich in jüngerer Zeit die Untersuchungen auf die Lösung dieses Problems konzentriert. Die bisherigen Ergebnisse zeigten, dass der Fettgehalt des Ganzkörpers bei Neugeborenen (MILISITS et al., 1999a) und Jungtieren (MILISITS et al., 2000) bzw. bei Muttertieren (SZENDRŐ et al., 1998) nur mit mittlerer Genauigkeit zu schätzen ist.

In vorliegenden Untersuchungen soll geprüft werden, ob ein Selektionsprogramm auf den Fettgehalt mit der TOBEC Methode durchführbar ist. Daher erfolgten:

1. Bestimmung des Körperfettgehaltes der Jungkaninchen im Alter von 10 Wochen anhand einer früher ausgearbeiteten Schätzungsgleichung. 
2. Anhand des geschätzten Fettgehaltes die Verwirklichung einer Selektionsstrategie zur Schaffung von zwei sich im Körperfettgehalt erheblich unterscheidenden Kaninchenpopulationen.

3. Vergleich einiger Reproduktionsmerkmale der Häsinnen und der Körperzusammensetzung von Nachkommen beider Populationen.

\section{Material und Methoden}

Die Untersuchungen wurden in der Versuchsanlage der Fakultät für Nutztierwissenschaften der Universität Kaposvar mit Tieren der Rasse Pannon-Weiße durchgeführt. Die Kaninchen wurden nach der Entwöhnung im Alter von 6 Wochen im Stall bei einer 16-stündigen Beleuchtung in Käfigen aus punktverschweißtem Drahtgitter (800x500mm) in Gruppen von 5-6 Kaninchen/Käfig gehalten. Als Futtermittel wurde das im Handel erhältliche Kaninchenfutter (DE $10.30 \mathrm{MJ} / \mathrm{kg}$, Rohprotein 17,5\%, Rohfett 3,6\%, Rohfaser 12,4\%) ad libitum verwendet. Trinkwasser stand den Kaninchen aus Selbsttränken ständig zur Verfügung.

Im Alter von 10 Wochen wurde der Versuchsbestand gewogen. Auf Grund des 10Wochen-Gewichtes und der Zunahmen zwischen der 6. und 10. Lebenswoche wurden die den Durchschnitt repräsentierenden Tiere (Durchschnitt \pm Abweichung) ausgewählt. Ihr Fettgehalt im Organismus wurde in lebendem Zustand mit dem Gerät EMSCAN SA-3152 bestimmt. Mit diesem Gerät wird das unterschiedliche elektrische Leitungsvermögen der in das elektrische Feld gesetzten ganzen Körper gemessen (Van LOAN und MAYCLIN, 1987). Die Messungen wurden in der Untersuchungseinheit in einem induzierten oszillierenden Magnetfeld, bei einer Frequenz von $10 \mathrm{Mhz}$, durchgeführt. Bei dieser Frequenz weicht die Leitfähigkeit des Fettgewebes vom fettfreien Gewebe ab, so dass auf Grund des mit dem Gerät gemessenen sogenannten E-Wertes das Gewicht ohne Fett gut geschätzt werden kann. Davon ausgehend kann dann das Fettgewicht errechnet werden.

Das Wesentliche der auf dem sogenannten Harker-Prinzip basierenden Untersuchungsmethode ist, dass zum Messen der Leitfähigkeit anstelle einer direkten elektrischen Verbindung die elektromagnetische Induktion benutzt werden kann. Die Methode dient somit streng genommen nicht der Messung der Leitfähigkeit, sondern der Messung der Energieabsorption im oszillierenden Magnetfeld.

Vom Prinzip der Messung ausgehend und wegen der Wechselwirkung zwischen dem Volumen des leitfähigen Körpers und dem sensorischen Magnetfeld, hat die geometrische Form des Untersuchungsobjektes sehr große Bedeutung (EM-SCAN MODEL SA-3000 OPERATOR'S MANUAL, 1996). Wegen der geringen Gewichtsunterschiede konnte von der schwierigen Querschnittsmessung abgesehen werden, da um störende Bewegungen auszuschließen, die Kaninchen während der Untersuchung in Bauchlage mit Gurten in gleicher Länge $(400 \mathrm{~mm})$ fixiert wurden. Jedes Tier wurde dreimal gemessen und für weitere Berechnungen das arithmetische Mittel verwendet. Das unmittelbare Ergebnis der Messung - der sogenannte E-Wert - wurde mit dem Programm SA3000 (läuft mit DOS 2.1), das vom Hersteller des TOBEC-Gerätes entwickelt wurde, bestimmt. Die Messabweichungen blieben bei allen Individuen unter $2 \%$. Die gemessenen Werte wurden in eine von MILISITS et al. (2000) erarbeitete Gleichung eingesetzt und damit der Fettgehalt berechnet.

Auf Grund dieser Schätzwerte wurden von den weiblichen Tieren jeweils die extrem $16 \%$, von den männlichen Tieren die extrem $8 \%$ fettesten bzw. magersten zur Wei- 
terzucht ausgewählt. Die Häsinnen wurden erstmalig im Alter von 17 Wochen mit frischem verdünnten Sperma besamt, das von Rammlern stammte, die nicht am Versuch teilnahmen. Das Ergebnis der Erstbesamung und des ersten Wurfes wurde nicht ausgewertet. $\mathrm{Ab}$ der zweiten Besamung, die 10 bis 11 Tage nach dem ersten Wurf erfolgte, wurde jede fette Mutter mit Sperma von fetten Rammlern besamt und umgekehrt. Verwendet wurde stets frisches, verdünntes Sperma. Folgende Wurfleistungsmerkmale wurden erfasst:

- $\quad$ Trächtigkeitsrate,

- $\quad$ notwendige Besamungen je Wurf,

- $\quad$ Anzahl der lebend- und totgeborenen Jungen je Wurfes,

- $\quad$ Wurfgewicht bei Geburt,

- $\quad$ Wurfgrösse und Wurfgewicht am 21. Lebenstag

- $\quad$ Verluste während der Säugezeit.

Der Fettgehalt der Nachkommen in der $F_{1}$ Generation wurde im Lebensalter von 10 Wochen mit der TOBEC Methode bestimmt und die den Durchschnitt repräsentierenden Tiere (Durchschnitt \pm Abweichung) gleich nach der Messung geschlachtet $(\mathrm{n}=22$ in der fetten und $n=20$ in der mageren Gruppe). Nach der Schlachtung wurde das Gewicht des Nacken- und Nierenfettes gewogen und ins Verhältnis zum Schlachtgewicht gesetzt.

Die Auswertung der Daten erfolgte mit der einfaktoriellen Varianzanalyse, der Diskriminanz-Analyse bzw. dem Chi ${ }^{2}$-Test. Für die statistischen Berechnungen wurde das Statistikprogramm SPSS for Windows genutzt (SPSS FOR WINDOWS, 1999).

\section{Ergebnisse}

Einige wichtige Parameter der zur Weiterzucht ausgewählten Tiere aus dem Versuchsbestand zeigt Tabelle 1.

Tabelle 1

Merkmale der auf Grund geschätzter Fettwerte zur Weiterzucht ausgewählten Kaninchen (Traits of the rabbits chosen for the experiment based on their fat content)

\begin{tabular}{c|c|c|c|c|c|c|c|c}
\hline Merkmal & \multicolumn{4}{|c|}{ fett } & \multicolumn{4}{|c}{ mager } \\
\hline & \multicolumn{2}{|c|}{ Mütter (n=63) } & \multicolumn{2}{|c|}{ Rammler (n=33) } & \multicolumn{2}{c}{ Mütter (n=57) } & \multicolumn{2}{c}{ Rammler (n=36) } \\
\hline & $\begin{array}{c}\text { Durch- } \\
\text { schnitt }\end{array}$ & $\begin{array}{c}\text { Abwei- } \\
\text { chung }\end{array}$ & $\begin{array}{c}\text { Durch- } \\
\text { schnitt }\end{array}$ & $\begin{array}{c}\text { Abwei- } \\
\text { chung }\end{array}$ & $\begin{array}{c}\text { Durch- } \\
\text { schnitt }\end{array}$ & $\begin{array}{c}\text { Abwei- } \\
\text { chung }\end{array}$ & $\begin{array}{c}\text { Durch- } \\
\text { schnitt }\end{array}$ & $\begin{array}{c}\text { Abwei- } \\
\text { chung }\end{array}$ \\
\hline $\begin{array}{c}\text { 10-Wochen- } \\
\text { Gewicht (g) }\end{array}$ & 2280 & 140 & 2346 & 191 & 2305 & 174 & 2277 & 166 \\
\hline Fett (\%) & 7,5 & 1,4 & 8,4 & 1,3 & 4,2 & 1,4 & 3,6 & 1,4 \\
\hline
\end{tabular}

Die Daten zeigen, dass die geschätzten Fettwerte der beiden extremen Tiergruppen sehr unterschiedlich sind. Bei den fetten und mageren Müttern bzw. Rammlern sind die beobachteten Unterschiede signifikant $(\mathrm{P} \leq 0,05)$.

Die Trächtigkeitsraten der Häsinnen beider Versuchsgruppen unterschieden sich signifikant $(\mathrm{P} \leq 0,05)$. So lag die Trächtigkeitsrate nach Erstbesamung der Gruppe I bei 75,0 $\%$, in Gruppe II bei 55,8.

In der Wurfgrösse bei Geburt (Abb. 1) betrug der Unterschied bei der Anzahl gesamt geborener Jungen zwischen den Gruppen 0,8 Tiere zugunsten der mageren Mütter. Signifikant war der Unterschied beim Anteil tot geborener Jungen zwischen den Gruppen I und II bei 4,5\% bzw. 10,2\%. 


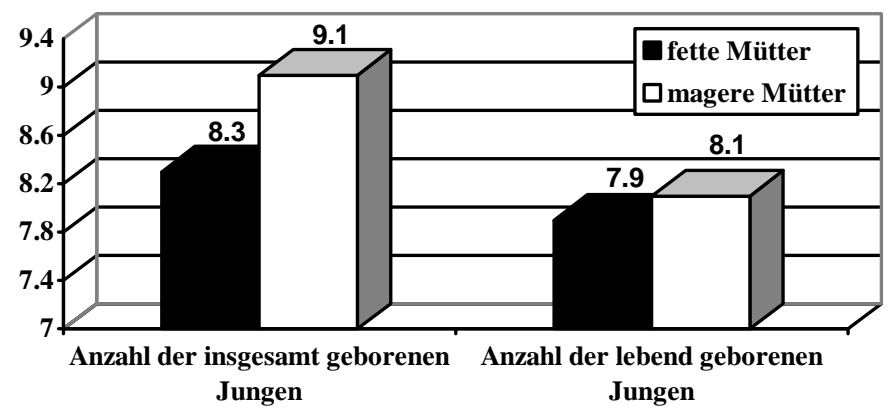

Abb. 1: Anzahl der insgesamt und der lebend geborenen Jungen bei fetten und mageren Müttern (Number of live born and total born pups of fatty and non-fatty does)

Die Verluste in der Säugezeit waren bei den fetten Müttern höher als in Gruppe II (Abb. 2). Die Unterschiede waren jedoch nicht signifikant.

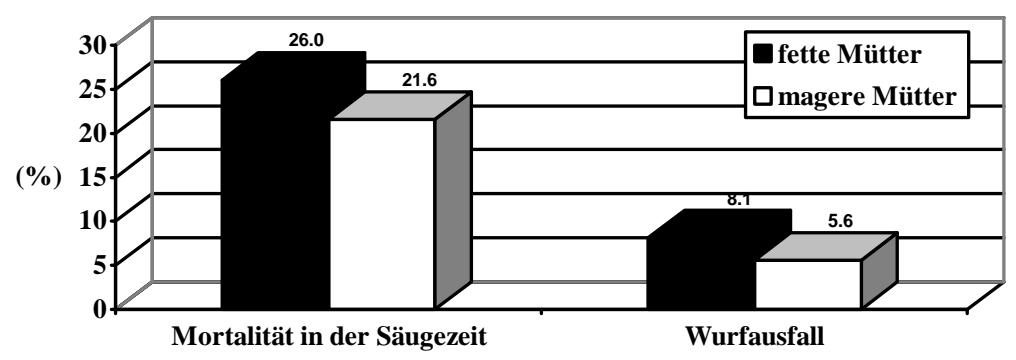

Abb. 2: Verluste und Wurfausfall in der Säugezeit bei fetten und mageren Häsinnen (Mortality and total litter loss at fatty and non-fatty does during the suckling period)

Wegen der unterschiedlichen Verluste in den Versuchsgruppen wurden sowohl in der Wurfgrösse am 21. Lebenstag als auch im 21 Tage Wurfgewicht bedeutende, aber statistisch nicht signifikante Unterschiede zwischen den Gruppen beobachtet.

Die TOBEC-Messungen in der $F_{1}$ Generation im Alter von 10 Wochen haben bei den Nachkommen der auf hohen Fettgehalt selektierten Kaninchen eine höhere Verfettung gezeigt (Abb. 3).

Bei den Nachkommen der auf hohen Fettgehalt ausgewählten Eltern überstiegen die geschätzten Fettgehalte die der Nachkommen von Gruppe II signifikant $(\mathrm{P} \leq 0,001)$ um $42 \%$ und betrugen $5,5 \%$ bzw. $3,8 \%$.

Die Ergebnisse der Ausschlachtungen haben die Unterschiede des Körperfettgehaltes in der $F_{1}$ Generation belegt. Es konnte eine signifikante Überlegenheit hinsichtlich eines engeren Verhältnisses des Nacken- und Nierenfettes zum Körpergewicht bei den von fetten Vorfahren stammenden Jungkaninchen nachgewiesen werden (Abb. 4).

Die in der auf hohen Fettgehalt selektierten Gruppe beobachteten Werte überstiegen beim Nackenfett um $26 \%$, beim Nierenfett um $51 \%$ die Werte der Gruppe mit niedrigen Fettgehalt.

Die Effektivität der Elternauswahl mittels der TOBEC-Methode wird durch die Tatsache bestätigt, dass die Nachkommen der auf geschätzten unterschiedlichen Fettgehalt beruhenden Elternauswahl, sich analog zu den Elterngruppen verhalten (Tab. 2). 


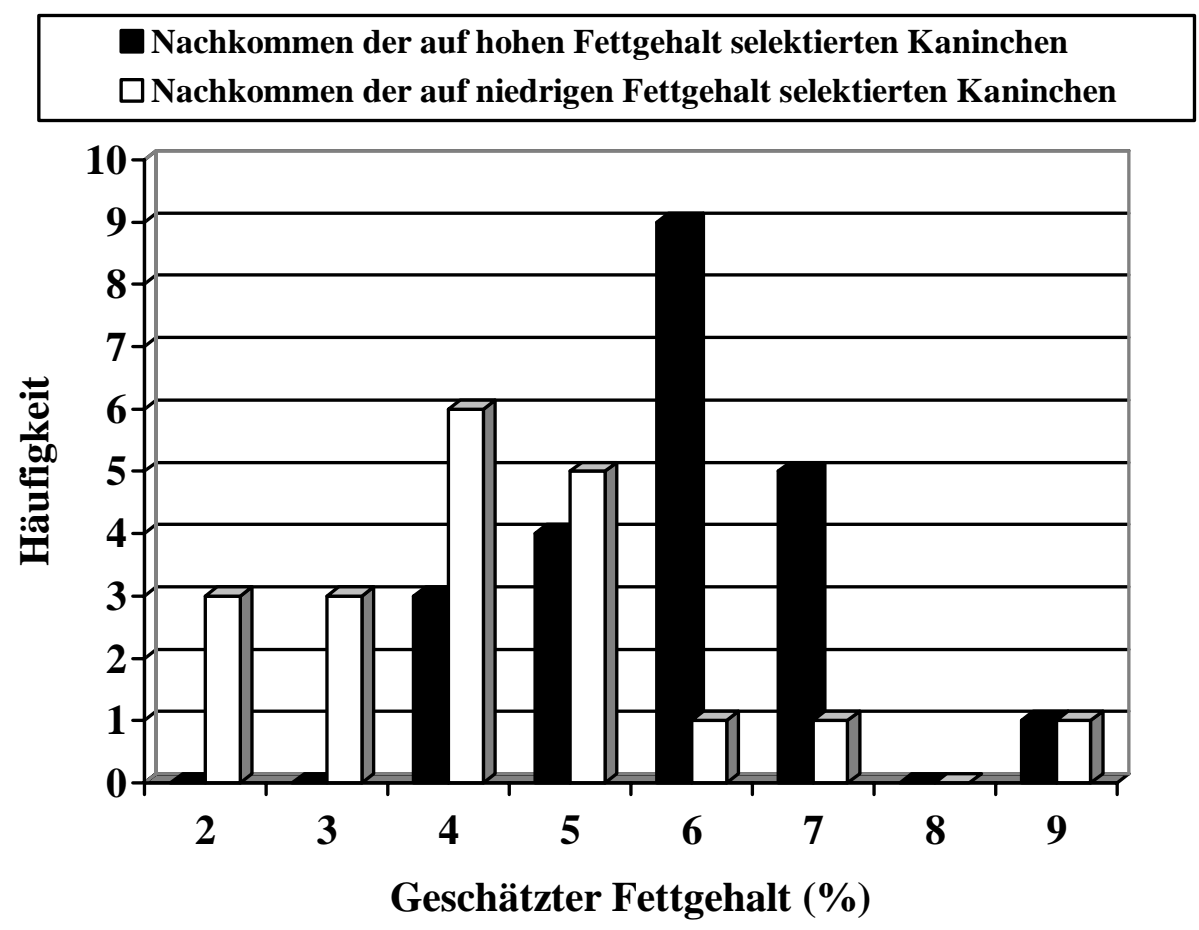

Abb. 3: Verteilung der Nachkommen von auf ihren Körperfettgehalt selektierten Kaninchen anhand der im Alter von 10 Wochen geschätzten Fettwerte (Histogram of the offsprings of fatty and non-fatty parents based on their fat content estimated at 10 weeks of age)

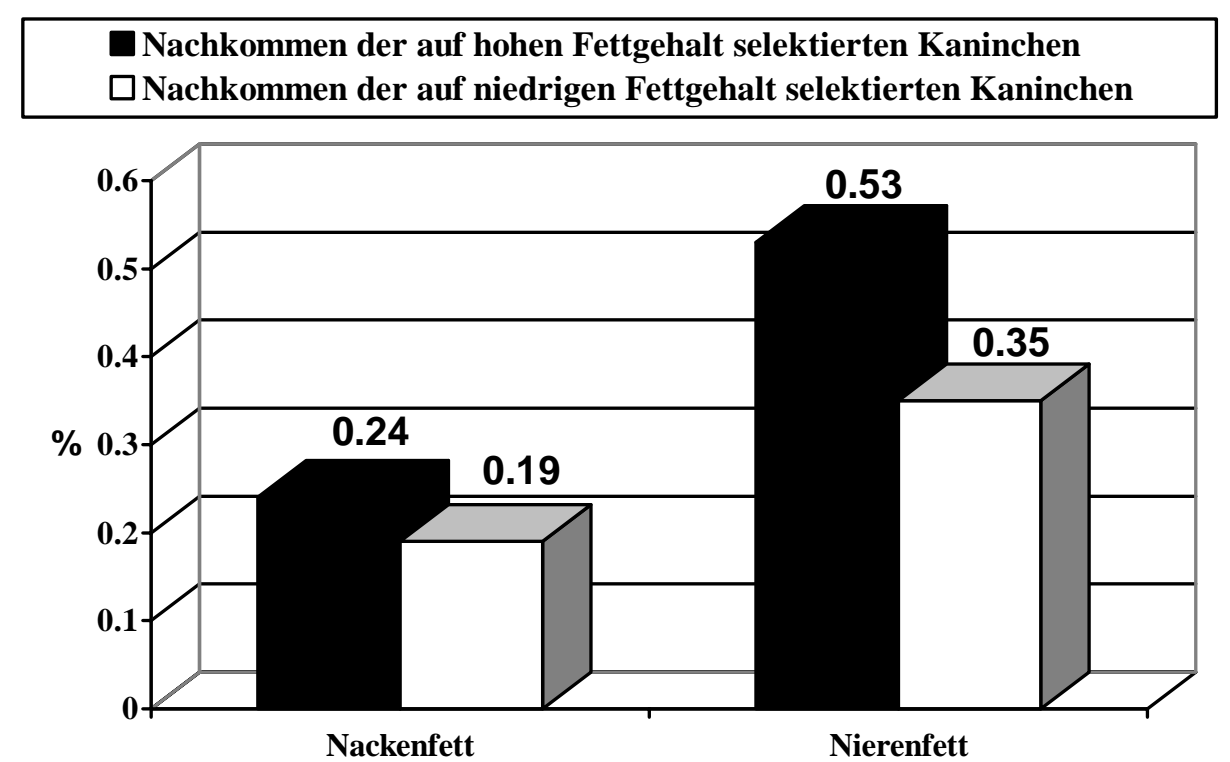

Abb. 4: Nacken- und Nierenfettgehalt der von fetten und mageren Vorfahren abstammenden Jungkaninchen im Alter von 10 Wochen (Ratio of the scapular and abdominal fat to the liveweight in the offsprings of fatty and non-fatty rabbits)

Tabelle 2

Diskriminant-Analyse anhand der geschätzten Fettwerte (Result of the discriminant analysis based on the fat content estimated)

\begin{tabular}{c|c|c}
\hline Originale Gruppen & \multicolumn{2}{|c}{ Geschätzte Gruppen } \\
\hline & fette Kaninchen & magere Kaninchen \\
\hline fette Kaninchen $(\mathrm{n}=22)$ & $81,8 \%(18)$ & $18,2 \%(4)$ \\
\hline magere Kaninchen $(\mathrm{n}=20)$ & $25 \%(5)$ & $75 \%(15)$ \\
\hline
\end{tabular}




\section{Diskussion}

Die Erklärung für den beobachteten Unterschied in der Trächtigkeitsrate zugunsten der fetteren Häsinnen kann der Energieverlust während der ersten Trächtigkeit und Laktation sein, der sich bei mageren Tieren negativ auswirken kann. Wie aus der Fachliteratur bekannt, brauchen die Häsinnen nach dem 20. Graviditätstag eine weitere Ergänzung an Energie und Nährstoffen, um eine gesunde Entwicklung der Föten zu sichern. Die Bedarfsdeckung ist aber nicht einfach, weil die immer intensivere - kurzfristig exponentielle, vom 23. Tag an nahezu lineare - Zunahme der Föten (PRUD'HON und SELME, 1973) die Futteraufnahmekapazität der Häsinnen immer mehr einschränkt. In dieser Phase der Gravidität steht also einem erhöhten Bedarf an Nährstoffen eine verminderte Futteraufnahme gegenüber. So ist eine Mobilisierung der maternalen Energiereserven - bei üblichem Energiegehalt des Futters von 10-12 MJ DE/kg und Wurfgrößen mit mehr als 4 Jungtieren - erforderlich (KAMPHUES, 1985). Während der ersten Trächtigkeit und Laktation kann der Energie- und Fettverlust 20 bis $40 \%$ betragen (PARIGI-BINI und XICCATO, 1993). Dadurch ist es wahrscheinlich, daß die mageren Mütter 10-11 Tage nach dem ersten Wurf nicht genug Reserven für die nächste Trächtigkeit haben. Das trifft vermutlich auch für die Anzahl der nötigen Besamungen für den nächsten Wurf zu. So waren bei den fetten Häsinnen durchschnittlich nur 1,26, bei den mageren jedoch 1,51 Besamungen für einen erfolgreichen Wurf notwendig.

Als Hintergrund des Unterschiedes im Anteil der tot geborenen Jungen kann wiederum den Energieverlust der Häsinnen während der ersten Gravidität angenommen werden. Die magere Mütter hatten wahrscheinlich nicht genug Reserven, um die nötigen Energie und Nährstoffen für alle Föten zu sichern, was die Anzahl der tot geborenen Jungen in dieser Gruppe vergrößerte.

Die beobachteten Unterschiede zwischen den geschätzten Fettwerten der Jungkaninchen stimmten mit den untersuchten Fettmerkmalen der geschlachteten Tiere überein. Das galt auch für den Vergleich der TOBEC-Schätzwerte aller 230 Nachkommen beim Vergleich beider Gruppen die im Alter von 10 Wochen untersucht wurden. Der Unterschied zwischen den beiden Gruppen war signifikant $(\mathrm{P} \leq 0.001)$.

Als Schlussfolgerung ist festzustellen, dass die TOBEC Methode zur Selektion der Kaninchen auf Grund des Fettgehaltes im Organismus verwendbar ist. Es konnte nachgewiesen werden, dass der Fettgehalt einen Einfluss auf die Wurfleistungen der Häsinnen hat. Im vorliegenden Versuch traf dies besonders für die Merkmale Trächtigkeitsrate, Wurfgrösse und insbesondere den Anteil tot geborener Jungen zu. Vorliegende Ergebnisse entstammen aus der ersten Phase eines Versuches und sind daher als vorläufig anzusehen. Im weiteren Versuchsablauf werden genetische Parameter sowie der Einfluss des Fettgehaltes im Tierkörper auf vermehrungsbiologische Eigenschaften in diesem Langzeitversuch weiter untersucht und darüber berichtet.

\section{Danksagung}

Die Forschung wurde vom Landesfonds für Wissenschaft und Forschung (OTKA F032594) und vom János Bolyai Forschungsstipendium unterstützt. 


\section{Literatur}

BENTSEN, H.B.; SEHESTED, E.; KOLSTAD, N.; KATLE, J.:

Body composition traits in broilers measured by computerised tomography. Proceedings of the Second International Poultry Breeders Conference and Artificial Insemination Workshop, Ayr (1986), 27-35

CUNNINGHAM, J.; MOLNAR, J.; MEARA, P.A.; BODE, H.H.:

In vivo total body electrical conductivity (TOBEC) following perturbations of body fluid compartments

EM-SCAN in rats. Metabolism, 35 (1986), 572-575

FEKETE, S.:

Model SA-3000 Operator's manual (1996)

The rabbit body composition: Methods of measurement, significance of its knowledge and the obtained results - a critical review. Journal Applied Rabbit Research, 15 (1992) 72-85

FEKETE, S.; BROWN, D.L.:

The major chemical components of the rabbit whole body measured by direct chemical analysis, deuterium oxide dilution and total body electrical conductivity. J. Vet. Nutr., 2 (1993) 23-29

FEKETE, S.; KÓSA, E.; ANDRÁSOFSZKY, E.; HULLÁR, I.:

In vivo measurements of body composition of dwarf and normal rabbit. $9^{\text {th }}$ Symposium on Housing and Diseases of Rabbits, Furbearing Animals and Fancy Pet Animals, Celle (1995), 223-234

FIOROTTO, M.L.; COCHRAN, W.J.; FUNK, R.C.; SHENG, H-P.; KLISH, W.J.:

Total body electrical conductivity measurements: effect of body composition and geometry. Am. J. Physiol. 252. (Regulatory Integrative Comp. Physiol. 21): (1987), 794-800

KALLWEIT, E.; WESEMEIER, H.H.; SMIDT, D.; BAULAIN, U.:

Einsatz der Magnet-Resonanz-Messung in der Tierzuchtforschung. Arch. Tierz., Dummerstorf 37 (1994) 2, 105-120

KAMPHUES, J.:

Untersuchungen zum Energie- und Nährstoffbedarf gravider Kaninchen. Züchtungskunde, 57 (1985) 3, $207-222$

KÖVÉR, GY.; SZENDRŐ, ZS.; ROMVÁRI, R.; JENSEN, J.F.; SØRENSEN, P.; MILISITS, G.:

In vivo measurement of body parts and fat deposition in rabbits by MRI. World Rabbit Science, 6 (1998) 2, 231-235p

MILISITS, G.:

Examination of changes in the body composition of growing rabbits and rabbit does by computer tomography and TOBEC method (in Hungarian). PhD thesis, Kaposvár, (1998), 124p

MILISITS, G.; GYARMATI, T.; SZENDRÖ, ZS.:

In vivo estimation of body fat content of new-born rabbits using the TOBEC method. World Rabbit Science, 7 (1999a ) 3, 151-154

MILISITS, G.; ROMVÁRI, R.; DALLE ZOTTE, A.; SZENDRŐ, ZS.:

Non-invasive study of changes in body composition in rabbits during pregnancy using X-ray computerized tomography. Annales de Zootechnie, 48 (1999b), 25-34

MILISITS, G.; SZENDRŐ, ZS.; MIHÁLOVICS, GY.; BIRÓ-NÉMETH, E.; RADNAI, I.; LÉVAI, A.:

Use of the TOBEC method for predicting the body composition of growing rabbits. $7^{\text {th }}$ World Rabbit Congress, Valencia, Vol. 1. (2000), 637-642

PARIGI-BINI, R.; XICCATO, G.:

Recherches sur l'interaction entre alimentation, reproduction et lactation chez la lapine, une revue. World Rabbit Science, 1 (1993) 4, 155-161

PRUD'HON, M.; SELME, M.:

Croissance ponderale des foetus et des placentas maternels et foetaux au cours de la gestation chez les lapines saillies post partum et chez des lapines temoins. Journées de recherches avicoles et cunicoles,

ROMVÁRI, R.: Publication de L'I.T.A.V.I., (1973), 51-54

Usefulness of computer tomography in the in vivo prediction of body composition and dressing percentage in rabbits and broiler chicks (in Hungarian). PhD thesis, Kaposvár, (1996)

ROSNER, F.; POLTEN, S.; WICKE, M.:

Vergleichsuntersuchungen zur Verwendbarkeit des PIGLOG-Ultraschall-Gerätes für die Vorausbestimmung des Muskelfleischanteils bei Sauen im Rahmen der Eigenleistungsprüfung. Arch. Tierz., Dummerstorf 43 (2000) 5, 499-506

SCHOLZ, A.; MITCHELL, A.D.; WANG, P.C.; SONG, H.; YAN, Z.:

Muscle metabolism and body composition of pigs with different ryanodine receptor genotypes studied by means of ${ }^{31} \mathrm{P}$ nuclear magnetic resonance spectroscopy and ${ }^{1} \mathrm{H}$ magnetic resonance imaging. Arch. Tierz., Dummerstorf 38 (1995) 5, 539-552

SKJERVOLD, H.; GRØNSETH, K.; VANGEN, O.; EVENSEN, A.: 
In vivo estimation of body composition by computerized tomography. Z. Tierz. Züchtungsbiologie. 98 (1981), 77-79

SPSS FOR WINDOWS:

Version 10.0, Copyright SPSS Inc. (1999)

STAUDINGER, F.B.; RORIE, R.P.; ANTHONY, N.B.:

Evaluation of a noninvasive technique for measuring fat-free mass in poultry. Poultry Science, $\mathbf{7 4}$ (1995), 271-278

SZENDRÖ, ZS.; BALLAY, A.; BIRÓNÉ, NÉMETH E.; MÁTYÁS, I.:

First results and experiences of the offsprings examinations in rabbit breeding (in Hungarian). Szaktanácsok, ATEK Kaposvár, 4 (1988), 26-29

SZENDRÖ, ZS.; HORN, P.; KÖVÉR, GY.; BERÉNYI, E.; RADNAI, I.; BIRÓNÉ, NÉMETH E.:

In vivo measurement of the carcass traits of meat type rabbits by X-ray computerised tomography. $5^{\text {th }}$ World Rabbit Congress, Corvallis; Journal Applied Rabbit Research, 15 (1992), 799-809

SZENDRÖ, ZS.; MILISITS, G.; ROMVÁRI, R.; LÉVAI, A.; GYARMATI, T.; RADNAI, I.; BIRÓNÉ, NÉMETH E.:

Examination of the body composition of rabbits by the TOBEC method. 1. Rabbit does (in Hungarian). $10^{\text {th }}$ Hungarian Conference on Rabbit Production, Kaposvár, (1998), 107-114

VANGEN, O.:

Evaluation of carcass composition of live pigs based on computed tomography. $35^{\text {th }}$ Annual Meeting of the EAAP, The Hague, Netherlands. (1984), Vol. 1. Summaries. Study Commissions. Genetics, nutrition, management. Paper No. G5. 23, 2

VAN LOAN, M.; MAYCLIN, P.:

A new TOBEC instrument and procedure for the assessment of body composition: use of Fourier coefficients to predict lean body mass and total body water. Am J. Clin Nutr., 45 (1987), 131-137

Eingegangen: 31.01 .2002

Akzeptiert: 03.07.2002

Anschrift der Verfasser

ANDRÁS LÉVAI, PhD Student

GÁBOR MILISITS, PhD

Universität Kaposvár, Fakultät für Nutztierwissenschaften

Guba S. u. 40.

H-7400 KAPOSVÁR

UNGARN 